\title{
CHAOTIC FUNCTIONS WITH ZERO TOPOLOGICAL ENTROPY
}

\author{
J. SMITAL
}

\begin{abstract}
Recently $\mathrm{Li}$ and Yorke introduced the notion of chaos for mappings from the class $C^{0}(I, I)$, where $I$ is a compact real interval. In the present paper we give a characterization of the class $M \subset C^{0}(I, I)$ of mappings chaotic in this sense. As is well known, $M$ contains the mappings of positive topological entropy. We show that $M$ contains also certain (but not all) mappings that have both zero topological entropy and infinite attractors. Moreover, we show that the complement of $M$ consists of maps that have only trajectories approximable by cycles. Finally, it turns out that the original $\mathrm{Li}$ and Yorke notion of chaos can be replaced by (an equivalent notion of) $\delta$-chaos, distinguishable on a certain level $\delta>0$.
\end{abstract}

1. Introduction. Let $I$ denote a compact real interval and $C^{0}(I, I)$ denote the set of continuous maps of $I$ into itself. Let $f \in C^{0}(I, I), \delta \geqslant 0$, and let $S \subset I$ be a set such that for any $x, y \in S, x \neq y$, and any periodic point $p$ of $f$ :

$$
\begin{aligned}
& \limsup _{n \rightarrow \infty}\left|f^{n}(x)-f^{n}(y)\right|>\delta, \\
& \liminf _{n \rightarrow \infty}\left|f^{n}(x)-f^{n}(y)\right|=0, \\
& \limsup _{n \rightarrow \infty}\left|f^{n}(x)-f^{n}(p)\right|>\delta .
\end{aligned}
$$

Here $f^{n}$ is the $n$th iterate of $f$. Then $S$ is called a scrambled set of $f$, or (when $\delta>0$ ) a $\delta$-scrambled set. The function $f$ is chaotic in the sense of Li and Yorke [11] if $f$ has an uncountable scrambled set. Any map with a cycle of order $\neq 2^{n}$ for $n=0,1,2, \ldots$ is chaotic in this sense (cf. [5]).

Recall that if $f$ has no cycles of order not a power of 2, then by Šarkovskii's theorem [14] (cf. also [24]) either $f$ has cycles of finitely many different orders, i.e., of orders $1,2, \ldots, 2^{k}$ for some $k$, or $f$ has a cycle of any of the orders $1,2,2^{2}, \ldots, 2^{n}, \ldots$ In the last case $f$ is referred to as a function of type $2^{\infty}$.

Functions with cycles of order not a power of 2 are often considered as the chaotic functions since they share a number of properties not possessed by other maps and since in some sense they behave more wildly. An extensive survey of the corresponding properties can be found, e.g., in [19 or 4]. Here we recall only some of them; they will be useful in the sequel.

1.1. THEOREM. Let $f \in C^{0}(I, I)$. The following conditions are equivalent:

(i) f has a cycle of order not a power of 2;

(ii) for some $x \in I$, the attractor $L_{f}(x)$ of $x$ is infinite and contains a periodic point (Šarkovskii [16, p. 71]; see also [17 or 19]);

1980 Mathematics Subject Classification. Primary 54H20, 26 A18.

Received by the editors July 12, 1985 and, in revised form, October 15, 1985. 
(iii) the topological entropy of $f$ is positive (Misiurewicz [12]);

(iv) there are closed intervals $J, K \subset I$ having at most one point in common, and positive integers $m, n$ such that $J \cup K \subset f^{m}(J) \cap f^{n}(K)$.

The equivalence of (i) and (iv) follows easily from [15 or 1] (see also [3]) where certain modifications of (iv) equivalent to (i) are given. However, (iv) seems to be more suitable in application.

The main aim of this paper is to show that functions chaotic in the sense of $\mathrm{Li}$ and Yorke need not have cycles of order not a power of 2 (Theorem 2.7 below). Moreover, we give a characterization of functions chaotic in this sense (Theorem 2.2). It turns out that the class $C^{0}(I, I)$ can be decomposed into two disjoint sets: Functions chaotic in the sense of $\mathrm{Li}$ and Yorke, and functions that have only trajectories approximable by cycles (Theorem 2.4). This shows that the Li and Yorke concept of chaos is a good and appropriate tool in considering maps of type $2^{\infty}$.

Since the proof of the above-quoted results is complicated, we first state in the next section the main results and in $\$ 3$ we prove some properties of functions of type $2^{\infty}$. Finally, in $\S 4$ we prove the main results, except for Theorem 2.7 ; its proof is given in $\$ 5$.

2. Main results. Let $f \in C^{0}(I, I)$ : Recall that the trajectory of $x \in I$ under $f$ is the sequence $\left\{x_{n}\right\}_{n=0}^{\infty}$, where $x_{0}=x$ and $x_{n+1}=f\left(x_{n}\right)$. The attractor $L_{f}(x)$ of $x$ is the set of limit points of the trajectory of $x$. An interval $O \subset I$ is $f$-periodic of order $k$ if $J, f(J), \ldots, f^{k-1}(J)$ are pairwise disjoint intervals and $f^{k}(J)=J$. For any set $A \subset I$ denote $\operatorname{Orb}_{f}(A)=\bigcup_{i=0}^{\infty} f^{i}(A)$. For $x \in I$ we write $\operatorname{Orb}_{f}(x)$ instead of $\operatorname{Orb}_{f}(\{x\})$. The Lebesgue measure is always denoted by $\mu$.

2.1. Definition. Let $f \in C^{0}(I, I)$. Then $u, v \in I$ are $f$-separable points if there are disjoint $f$-periodic intervals $J_{u}, J_{v} \subset I$ with $u \in J_{u}, v \in J_{v}$. Otherwise $u, v$ are $f$-nonseparable.

2.2. Theorem. Let $f \in C^{0}(I, I)$. Assume $f$ has zero topological entropy. Then the following conditions are equivalent:

(i) There is an infinite attractor $L_{f}(x)$ for some $x \in I$ containing two f-nonseparable points.

(ii) For some $\delta>0$, f has a nonempty perfect $\delta$-scrambled set.

2.3. Remark. Condition 2.2(ii) implies that $f$ is topologically conjugate to a function $g \in C^{0}(I, I)$, which has a scrambled set of positive Lebesgue measure. To see it, let $S$ be a perfect scrambled set for $f$, and let $\varphi$ be a homeomorphism of $I$ such that $\mu(\varphi(S))>0$. Then clearly $S^{*}=\varphi(S)$ is a scrambled set for $g=\varphi \circ f \circ \varphi^{-1}$. Moreover, when $S$ is $\delta$-scrambled, then also $S^{*}$ can be made to be $\delta$-scrambled. Thus we have a result generalizing recent results from [13, 7, 21 and 22]. Note that in all these papers the corresponding chaotic functions have positive topological entropy.

2.4. THEOREM. Let $f \in C^{0}(I, I)$ be of type $2^{\infty}$ and let no infinite attractor of $f$ contain two f-nonseparable points. Then for any $x \in I$ and $\varepsilon>0$ there is a periodic 
point $p$ of $f$ with

$$
\limsup _{n \rightarrow \infty}\left|f^{n}(x)-f^{n}(p)\right|<\varepsilon .
$$

2.5. Remark. Condition 1.1(iv) allows us to prove that any $f \in C^{0}(I, I)$ with positive topological entropy has a nonempty perfect $\delta$-scrambled set for certain $\delta>0$. The argument uses some ideas from Misiurewicz's paper [13] and can be found in [23]. Thus by Theorem 2.4, condition 2.2(ii) gives a characterization of maps from $C^{0}(I, I)$, chaotic in the sense of $\mathrm{Li}$ and Yorke.

2.6. REMARK. An arbitrarily small perturbation of a nonchaotic mapping can provide a chaotic map, but in certain cases, the chaos can only be small, cf. [20, 25]. The result is as follows: If $f \in C^{0}(I, I)$ has zero topological entropy, and the set of periodic points is closed and nowhere dense, then for any given $\varepsilon>0$, every $g \in C^{0}(I, I)$ sufficiently near to $f$ has only trajectories $\varepsilon$-approximable by cycles. In connection with the above results, we conjecture that this condition can be extended to nonchaotic maps $f \in C^{0}(I, I)$, whose periodic points form a nowhere dense set, i.e. also for certain maps with infinite attractors (the set of periodic points of any such mapping cannot be closed, cf. e.g. [15]).

2.7. THEOREM. (i) There is a function $f \in C^{0}([0,1],[0,1])$ of type $2^{\infty}$ which is chaotic in the sense of Li and Yorke.

(ii) There is a map $g \in C^{0}([0,1],[0,1])$ of type $2^{\infty}$ which has an infinite attractor, but is not chaotic in the sense of Li and Yorke.

3. Properties of functions of type $2^{\infty}$. The following theorem summarizing certain known results will be useful in the sequel.

3.1. Theorem. Assume $f \in C^{0}(I, I)$ is of type $2^{\infty}$ and $\omega$ is its infinite attractor. Then

(i) $f(\omega)=\omega$;

(ii) any neighborhood of an arbitrary nonisolated point $x \in \omega$ contains a periodic point of $f$

(iii) $\omega=A \cup B$, where $A \neq \varnothing$ is nowhere dense and perfect and $B$ is countable with the property that every interval contiguous to $A$ contains at most two points from $B$.

Property (i) is well known and easily provable for any $f \in C^{0}(I, I)$, (iii) is proved in [18]. Condition (ii) is proved under more general assumptions in [8]; however, since [8] is not generally available, we later give an independent proof (Remark 3.6).

For simplicity, we now state some standard assumptions and notation that we will use in this section.

3.2. Assumptions. Let $f \in C^{0}(I, I)$ be of type $2^{\infty}$. Let $\omega=L_{f}(x)$ be an infinite attractor and let $\left\{x_{i}\right\}_{i=0}^{\infty}$ be the trajectory of $x$. Put $a=\min \omega, b=\max \omega$, and let $c \in(a, b)$ be a fixed point of $f$, i.e., $c=f(c)$; the existence of $c$ follows from 3.1(i) since by $1.1(\mathrm{ii})$

$$
f(y) \neq y \quad \text { for every } y \in \omega .
$$


3.3. Lemma. Let Assumptions 3.2 be satisfied. Then for any sufficiently large $i$, $x_{i}<c$ iff $x_{i+1}>c$.

Proof. By (3.1) and 3.1(i) there is an interval $J=[u, v] \subset(a, b)$ with $c \in J$ and $f^{2}(J) \supset[a, b]$. (If $f(a)=b$, take $a_{1}, a_{2} \in \omega$ with $f\left(a_{2}\right)=a_{1}, f\left(a_{1}\right)=a$ and put $J=\operatorname{conv}\left\{a_{1}, a_{2}, c\right\}$, the convex hull of $\left\{a_{1}, a_{2}, c\right\}$.)

Denote $f^{2}([a, c])=J_{0}, f^{2}([c, b])=J_{1}$. We show that for some $m, n$ there are $x_{m} \in J_{0}$ and $x_{n} \in J_{1}$. Indeed, if $x_{i} \notin[a, c]$ for every $i$, then $(a, c) \cap \omega=\varnothing$; so $f(a), f^{2}(a)>c$ (see 3.1(i)) and since by (3.1) $f(a) \neq f^{2}(a)$, we have either $(c, f(a))$ $\cap \omega \neq \varnothing$ or $\left(c, f^{2}(a)\right) \cap \omega \neq \varnothing$. In any case, $x_{m} \in f((c, f(a))) \subset f^{2}([a, c])=J_{0}$ for some $m$. Similarly for $J_{1}$.

Now assume the lemma is not true. Then there is a $k>m+n$ with

$$
x_{k}, x_{k+1}<c<x_{k+2} \text { or } x_{k+2}<c<x_{k}, x_{k+1} \text {. }
$$

A simple verification shows that in any case, for some $s \in\{1,2\}$,

$$
f^{s}\left(\operatorname{conv}\left\{c, x_{k}\right\}\right) \supset \operatorname{conv}\left\{x_{k+1}, x_{k+2}\right\} \text {. }
$$

Moreover, there is an $r>0$ with

$$
f^{r}\left(\operatorname{conv}\left\{x_{k+1}, x_{k+2}\right\}\right) \supset[u, v] .
$$

To see it assume, e.g., $x_{k+1}<c<x_{k+2}$. If $u<x_{k+1}$, choose $p>0$ such that $x_{k+p+1}<u$. If $x_{k+1}<u$, then clearly there are $p>0$ and $t$ with $x_{k+1}<x_{t}<c$ and $x_{t+p}<\min \left\{x_{t}, u\right\}$ (note that $a=\min \omega<u$ ). In any case, $f^{p i}\left(\left[x_{k+1}, c\right]\right) \supset[u, c]$ for every $i>0$. Similarly, there is some $q>0$ such that $f^{q j}\left(\left[c, x_{k+2}\right]\right) \supset[c, v]$ for any $j>0$. Put $r=p q$.

By (3.2) and (3.3) we now have

$$
f^{2+k-m+s+r+2}([a, c]) \supset f^{s+r+2}\left(\operatorname{conv}\left\{x_{k}, c\right\}\right) \supset f^{2}([u, v])=[a, b] .
$$

Similarly, $f^{2+k-n+s+r+2}([c, b]) \supset[a, b]$ and Theorem 1.1 (see (iv)) gives a contradiction.

3.4. Lemma. Let Assumptions 3.2 be satisfied. Then there is an f-periodic interval $J \subset I$ of order 2 such that

$$
J \cap \omega \neq \varnothing \neq f(J) \cap \omega \text { and } \operatorname{Orb}_{f}(J) \supset \omega .
$$

Proof. Put $\omega_{0}=\{x \in \omega ; x<c\}$ and $\omega_{1}=\{x \in \omega ; x>c\}$. By (3.1), $\omega=\omega_{0} \cup$ $\omega_{1}$. Moreover, $f\left(\omega_{0}\right) \subset \omega_{1}$, since otherwise $f(y) \in \omega_{0}$ for some $y \in \omega_{0}$, and by the continuity of $f$ we have $x_{k}, x_{k+1}<c$ for a certain $k$, contrary to Lemma 3.3. Similarly, $f\left(\omega_{1}\right) \subset \omega_{0}$, and by $3.1(\mathrm{i})$

$$
f\left(\omega_{0}\right)=\omega_{1} \text { and } f\left(\omega_{1}\right)=\omega_{0} .
$$

Let $J=\bigcup_{i=1}^{\infty} f^{2 i}\left(\operatorname{conv} \omega_{0}\right), K=\bigcup_{i=1}^{\infty} f^{2 i}\left(\operatorname{conv} \omega_{1}\right)$. Since $f^{2}\left(\operatorname{conv} \omega_{i}\right) \supset \operatorname{conv} \omega_{i}$ for $i=0,1$, we have

$$
f^{2}(J)=J \text { and } f^{2}(K)=K
$$

Moreover, by (3.4),

$$
f(J)=\bigcup_{i=1}^{\infty} f^{2 i}\left(f\left(\operatorname{conv} \omega_{0}\right)\right) \supset \bigcup_{i=1}^{\infty} f^{2 i}\left(\operatorname{conv} \omega_{1}\right)=K
$$

and similarly, $f(K) \supset J$. Now (3.5) implies $f(J)=K$ and $f(K)=J$. 
To finish the proof it suffices to show that $c \notin J \cup K$ (this would imply $J \cap K=\varnothing$ ). Assume, e.g., that $c \in J$. Let $d \in \omega_{0}$ be the point nearest to $c$, and put $I_{1}=\operatorname{conv}\left\{\omega_{0}, c\right\}, I_{2}=\operatorname{conv} \omega_{0}, I_{3}=\operatorname{conv}\{d, c\}$. Since $c \in f^{s}\left(I_{2}\right)$ for some $s$, we have $f^{k}\left(I_{2}\right) \supset I_{1}$ for some $k$. Moreover, since $f^{2}(d)<d$ (see (3.1) and (3.4)), $f^{2}\left(I_{3}\right)$ contains some $x_{i}$ and, similarly, as in the proof of the preceding lemma we show that $f^{j}\left(\operatorname{conv}\left\{x_{i}, c\right\}\right) \supset[a, c]$ for some $j$. Thus $f^{k}\left(I_{2}\right) \supset I_{1}$ and $f^{j+2}\left(I_{3}\right) \supset I_{1}$, and Theorem 1.1 (see (iv)) gives a contradiction.

Now we are able to give one of the most important results in this section. It is implicitly contained in several Šarkovskii papers, but no correct reference can be given.

3.5. THEOREM. Let $f \in C^{0}(I, I)$ be of type $2^{\infty}$ and let $\omega$ be an infinite attractor of $f$. Then there is a sequence $\left\{J_{k}\right\}_{k=1}^{\infty}$ of $f$-periodic intervals with the following properties: For any $k$,

(i) $J_{k}$ has period $2^{k}$;

(ii) $J_{k+1} \cup f^{2^{k}}\left(J_{k+1}\right) \subset J_{k}$;

(iii) $\omega \subset \operatorname{Orb}_{f}\left(J_{k}\right)$;

(iv) $\omega \cap f^{i}\left(J_{k}\right) \neq \varnothing$ for every $i$.

The proof is induction from Lemma 3.4.

3.6. RemarK. Theorem 3.5 implies 3.1(ii). Indeed, let $U \subset I$ be a maximal interval containing no periodic points of $f$, and such that $U \cap \omega$ is infinite. Then at least one of the endpoints of $U$, say point $u$, lies in $\operatorname{Int}(\operatorname{conv} \omega)$. Hence $u$ is periodic of period $2^{k}$ for some $k$. Now using Theorem 3.5 find an $f$-periodic interval $J$ of order $m>2^{k}$ with $\bar{J} \subset \operatorname{Int}(U)$, where $\bar{J}$ denotes the closure of $J$. Clearly $f^{m}(\bar{J})=\bar{J}$, so $\operatorname{Int}(U)$ contains a periodic point - a contradiction.

3.7. Lemma. Let Assumptions 3.2 be satisfied and let $J$ be an interval with $\omega \cap J$ infinite. Then for some $k$ there is an f-periodic interval $K$ of order $2^{k}$ with $K \subset J$ and $\omega \subset \operatorname{Orb}_{f}(K)$.

Proof. By Theorem 3.1(ii) and (iii) there are periodic points $p, q \in J$ of order $\leqslant 2^{s}$, $p<q$, such that $(p, q) \cap \omega \neq \varnothing$. Let $U$ be any periodic interval of order $>2^{s}$ with $\omega \subset \operatorname{Orb}_{f}(U)$. Then clearly $p, q \in \operatorname{Orb}_{f}(U)$ and hence $K=f^{i}(U) \subset J$ for some $i$.

3.8. Lemma. Let Assumptions 3.2 be satisfied, and let $u<v$ be f-nonseparable points from $\omega$. Then $(u, v) \cap \omega=\varnothing$.

Proof. By Lemma 3.7, $(u, v) \cap \omega$ is finite (see also Theorem 3.5). Assume $z \in(u, v) \cap \omega$, and let $L$ be the maximal closed interval with $z \in L$ and $L \cap \omega$ finite (see 3.1(iii)). Clearly $u, v \in L$. Let $x_{n} \in(u, v)$. By Theorem 1.1 (see (ii)) there is $f^{k}(\{u, v\}) \cap L=\varnothing$ and $x_{n+k} \in(u, v)$ for some $k$. Put $U=\operatorname{conv}\left\{f^{k}(u), x_{n+k}\right\}$, $V=\operatorname{conv}\left\{f^{k}(v), x_{n+k}\right\}$. Then both $U \cap \omega$ and $V \cap \omega$ are infinite and by Lemma 3.7 there are $f$-periodic intervals $J_{1} \subset U$ and $J_{2} \subset V$ of certain order with $\operatorname{Orb}_{f}\left(J_{1}\right)$ $\cap \operatorname{Orb}_{f}\left(J_{2}\right) \supset \omega$. Choose $p, q$ such that $u, v \in f^{p}\left(J_{1}\right) \cap f^{q}\left(J_{2}\right)$. Then

$$
f^{k+p}\left(\left[u, x_{n}\right]\right) \cap f^{k+q}\left(\left[x_{n}, v\right]\right) \supset[u, v] .
$$

and Theorem 1.1 (see (iv)) gives a contradiction. 
The following result is the main tool in the proof of Theorem 2.2 .

3.9. Lemma. Let $f \in C^{0}(I, I)$ be of type $2^{\infty}$, and let $\omega$ be an infinite attractor of $f$ containing $f$-nonseparable points $u<v$. Let $U, V$ be disjoint connected neighborhoods of $u$ and $v$, respectively, such that $\operatorname{conv}(U \cap V)$ contains an $f$-periodic interval $J$ with $\operatorname{Orb}_{f}(J) \supset \omega$ and $u, v \in J$.

Then for any positive integer $q$ there is some $n$ divisible by $2^{q}$ such that $f^{n}(U) \cup f^{n}(V)$ contains an f-periodic interval $J^{*}$ with $\operatorname{Orb}_{f}\left(J^{*}\right) \supset \omega$ and $u, v \in J^{*}$.

Proof. By 3.1(ii) one of the sets $U, V$, say $U$, contains a periodic point $p$ of $f$ of order $2^{k}$. Let $J_{1} \subset J$ be a periodic interval of order $m=2^{s}$, where $s>\max \{k, q\}$, such that $u, v \in J_{1}$ and $\operatorname{Orb}_{f}\left(J_{1}\right) \supset \omega$ (Theorem 3.5). Denote $g=f^{m}, \omega_{1}=J_{1} \cap \omega$. Then $p$ is a fixed point of $g$ and $\omega_{1}=L_{g}(y)$ for some $y$. Note that $p \in U$ is to the left of $J_{1}$.

First we show that

$$
g^{i}(U) \supset J_{1} \text { for every } i \geqslant 2 .
$$

Let $J_{2} \subset J_{1}$ be a $g$-periodic interval of order 2 with $\operatorname{Orb}_{g}\left(J_{2}\right) \supset \omega_{1}$ and such that $J_{2}$ is to the left of $g\left(J_{2}\right)$. Then there is some $z \in U \cap J_{2} \neq \varnothing$ and $g^{i}(U) \supset$ $g^{i}(\operatorname{conv}\{p, z\}) \supset g^{i-1}\left(\operatorname{conv}\left\{p, J_{2}\right\}\right) \supset g^{i-2}\left(\operatorname{conv}\left\{p, g\left(J_{2}\right)\right\}\right) \supset g^{i-2}\left(J_{1}\right)=J_{1}$.

Now by (3.6) and Lemma 3.7 it suffices to show that for some $j, g^{j}(V) \cap \omega_{1}$ is infinite. So assume $g(V) \cap \omega_{1}$ is finite. Then $y_{r}=g^{r}(y) \in V$ for some $r$ and by Theorems 1.1(ii) and 3.1(iii) there is an $s$ with $y_{r+s} \in V$ and $g^{s}(v) \in U$ such that $\left(g^{s}(v), y_{r+s}\right) \cap \omega_{1}$ is infinite.

4. Proofs of the main results. We need some lemmas. First the notation. For any positive integer $k$, let $X(k)=\{0,1\}^{k}$ be the set of ordered $k$-tuples of two symbols and let $X=\{0,1\}^{N}$ be the set of infinite sequences of such type. If $\alpha \in X(k)$ and $\beta \in X(s)$, then $\alpha \beta \in X(k+s)$ is the concatenation of $\alpha$ and $\beta$. Assume $X$ is equipped with the topology of pointwise convergence.

4.1. Lemma. Let $f \in C^{0}(I, I)$ be of type $2^{\infty}$ and let $\omega$ be an infinite attractor of $f$ containing $f$-nonseparable points $u<v$.

Then there are a system $\left\{A_{\alpha} ; \alpha \in X(k)\right\}_{k=1}^{\infty}$ of closed subintervals of $I$ and an increasing sequence $\{n(k)\}_{k=1}^{\infty}$ of positive integers such that, for any $k$,

$$
\begin{array}{ll}
A_{\alpha \beta} \subset A_{\alpha} & \text { whenever } \alpha \beta \in X(k), \\
A_{\alpha} \cap A_{\beta}=\varnothing & \text { for } \alpha, \beta \in X(k), \alpha \neq \beta, \\
\text { if } \alpha \in X(k) & \text { then } f^{n(k)}\left(A_{\alpha}\right)=\bar{J}_{k},
\end{array}
$$

where $J_{k}$ is an f-periodic interval of order $\geqslant 2^{k}$ with $\operatorname{Orb}_{f}\left(J_{k}\right) \supset \omega$ and $u, v \in J_{k}$,

$$
n(k)-n(i) \text { is divisible by } 2^{i} \text {, for every } i<k
$$

and

$$
f^{n(k)}\left(A_{\alpha 0}\right) \subset(-\infty, u+1 / k) \text { and } f^{n(k)}\left(A_{\beta 1}\right) \subset(v-1 / k, \infty)
$$

for $\alpha, \beta \in X(k)$. 
Proof. Let $U_{0}^{n}, U_{1}^{n}$ be closed intervals with $u \in U_{0}^{n}, v \in U_{1}^{n}$, $\operatorname{conv}\left(U_{0}^{n} \cup U_{1}^{n}\right)=I$, and

$$
U_{0}^{n} \subset(-\infty, u+1 / n) \text { and } U_{1}^{n} \subset(v-1 / n, \infty)
$$

for $n=1,2, \ldots$ (see Lemma 3.8). Choose $n$ (1) such that

$$
f^{n(1)}\left(U_{0}^{1}\right) \cap f^{n(1)}\left(U_{1}^{1}\right) \supset \bar{J}_{1},
$$

where $J_{1}$ is an $f$-periodic interval of order $\geqslant 2$ with $u, v \in J_{1}$ and $\omega \in \operatorname{Orb}_{f}\left(J_{1}\right)$ (Lemma 3.9; note that any periodic interval contains the closure of a periodic interval). Let $A_{i} \subset U_{i}^{1}$ be a closed interval such that $f^{n(1)}\left(A_{i}\right)=\bar{J}_{1}$ for $i=0,1$.

Now assume by induction that $\left\{A_{\alpha} ; \alpha \in X(i)\right\}_{i=1}^{s}$ and $\{n(i)\}_{i=1}^{s}$ are defined in such a way that (4.1)-(4.5) hold for any $k \leqslant s$. By Lemma 3.9 we can choose a positive integer $m$ divisible by $2^{s}$ and such that

$$
f^{m}\left(U_{0}^{s+1} \cap J_{s}\right) \cap f^{m}\left(U_{1}^{s+1} \cap J_{s}\right) \supset \bar{J}_{s+1},
$$

where $J_{s+1}$ is an $f$-periodic interval of order $\geqslant 2^{s+1}$ with $u, v \in J_{s+1}$ and $\omega \subset$ $\operatorname{Orb}_{f}\left(J_{s+1}\right)$. Hence there is a closed interval $F_{i} \subset U_{i}^{s+1} \cap J_{s}$ such that $f^{m}\left(F_{i}\right)=\bar{J}_{s+1}$ and since (4.3) is true for $k=s$, we can find, for every $\alpha \in X(s)$, a closed interval $A_{\alpha i} \subset A_{\alpha}$ with $f^{n(s)}\left(A_{\alpha i}\right)=F_{i}, \quad i=0,1$. Put $n(s+1)=n(s)+m$. Clearly, the system $\left\{A_{\alpha} ; \alpha \in X(s+1)\right\}$ and $n(s+1)$ satisfy (4.1)-(4.5) for $k=s+1$ ((4.5) follows from (4.6)).

Let $\xi$ be an irrational number and define $\psi:[0,1] \rightarrow X$ as follows:

$$
\psi(t)=\left\{t_{n}\right\}_{n=1}^{\infty}, \quad \text { where } t_{n}= \begin{cases}0 & \text { if } E(t+n \xi) \in[0,1 / 2), \\ 1 & \text { if } E(t+n \xi) \in[1 / 2,1) .\end{cases}
$$

Here $E(x) \in[0,1)$ denotes the noninteger part of $x$.

4.2. Lemma. Let $\psi(s)=\left\{s_{n}\right\}_{n=1}^{\infty}$ and $\psi(t)=\left\{t_{n}\right\}_{n=1}^{\infty}$. Then

$$
t_{n} \neq s_{n} \quad \text { for infinitely many } n \text {, whenever } t \neq s \text {; }
$$

$\psi$ is a Borel map.

Proof. Property (4.7) follows easily from the fact that the sequence $\{E(n \xi)\}_{n=1}^{\infty}$ is uniformly distributed (and hence dense) in [0,1], see e.g. [9]. To prove (4.8) note that for any $n$ there is at most one $t=t(n) \in[0,1]$ with $t_{n}=1 / 2$ and that $\psi$ is continuous on $[0,1] \backslash\{t(n)\}_{n=1}^{\infty}$.

From the following lemma, (i) $\Rightarrow$ (ii) of Theorem 2.2 follows. However, the lemma gives a somewhat stronger result.

4.3. Lemma. Let the assumptions of Lemma 4.1 be satisfied. Then $f$ has a perfect nonempty $\delta$-scrambled set $S$, where $\delta=v-u$.

Proof. For any $k$, let $F_{k}=\bigcup\left\{A_{\alpha} ; \alpha \in X(k)\right\}$, where $A_{\alpha}$ are the sets from Lemma 4.1, and let $F=\bigcap_{k=1}^{\infty} F_{k}$. Then by (4.1) and (4.2), $F$ is a nonempty perfect set. Moreover, let $\left\{K_{i}\right\}_{i \in M}$ be the connected components of $\operatorname{Int}(F)$ and let $K_{i}=\left(x_{i}, y_{i}\right)$ for any $i$. Put $K_{i}^{*}=\left(x_{i}, y_{i}\right]$ and denote

$$
B=F \backslash \bigcup_{i \in M} K_{i}^{*} \text {. }
$$


Clearly, $B$ is an uncountable Borel set and there is a 1-1 map $\varphi$ of $X$ onto $B$ defined as follows: For $\alpha=\{\alpha(i)\}_{i=1}^{\infty}$ let $\varphi(\alpha) \in\left(\bigcap_{k=1}^{\infty} A_{\alpha(1) \cdots \alpha(k)}\right) \cap B$.

Now let $\psi$ be the map from Lemma 4.2. Since $\psi$ is $1-1,(4.8)$ implies that $\psi([0,1])$ is an uncountable analytic set. Hence there is an uncountable compact set $P \subset$ $\psi([0,1])$ (cf. [10, p. 387]). Moreover, since the set $X^{*} \subset X$ of sequences, which are eventually stationary, is countable, we may assume

$$
P \subset X \backslash X^{*} \text {. }
$$

Now since $\varphi^{-1}$ is clearly continuous and $1-1, \varphi(P) \subset[0,1]$ is an uncountable compact set. Hence there is a nonempty perfect set $S \subset \varphi(P)$.

It suffices to show that $S$ is a $\delta$-scrambled set for $f$, where $\delta=v-u$. Let $x \neq y$, $x, y \in S$. Denote $\varphi^{-1}(x)=\{x(i)\}_{i=1}^{\infty}, \varphi^{-1}(y)=\{y(i)\}_{i=1}^{\infty}$. By (4.7) there is an arbitrary large $j$ with $x(j) \neq y(j)$. Hence by (4.5),

$$
\begin{aligned}
\left|f^{n(j-1)}(x)-f^{n(j-1)}(y)\right| & \geqslant \operatorname{dist}\left\{f^{n(j-1)}\left(A_{x(1) \cdots x(j)}\right), f^{n(j-1)}\left(A_{y(1) \cdots y(j)}\right)\right\} \\
& >\delta-2 /(j-1)
\end{aligned}
$$

and thus (1.1) is true.

For any $k$ there are $\alpha, \beta \in X(k)$ such that $x \in A_{\alpha}, y \in A_{\beta}$, so by (4.3), $\left\{f^{n(k)}(x), f^{n(k)}(y)\right\} \subset \bar{J}_{k}$ and since $J_{k}$ is periodic of order $2^{k}$, there is some $m(k)$ with $0 \leqslant m(k)<2^{k}$ such that $\operatorname{diam}\left(f^{m(k)}\left(J_{k}\right)\right) \leqslant 2^{-k} \operatorname{diam}(I)$. Hence $\left|f^{n(k)+m(k)}(x)-f^{n(k)+m(k)}(y)\right| \leqslant 2^{-k} \operatorname{diam}(I)$ and this implies (1.2).

Finally, let $p$ be a periodic point of order $2^{r}$ and let $x \in S$. By (4.4), for any $k>r$,

$$
f^{n(k)}(p)=f^{n(r)}\left(f^{n(k)-n(r)}(p)\right)=f^{n(r)}(p)=q .
$$

Since the system $\left\{f^{i}\left(J_{r+1}\right)\right\}_{i=1}^{\infty}$ contains more than $2^{r}$ pairwise disjoint intervals, we have $q \notin \bar{J}_{r+1}$. By symmetry we may assume $q$ is to the left of $\bar{J}_{r+1}$, i.e., $q<u$. Let $\varphi^{-1}(x)=\{x(i)\}_{i=1}^{\infty}$. By (4.9) there is an arbitrarily large $s>r+1$ with $x(s)=1$. Hence $x \in A_{x(1) x(2) \cdots x(s-1) 1}$ and by (4.5), $f^{n(s-1)}(x)>v-1 /(s-1)$. Consequently, using (4.10), we have

$$
\left|f^{n(s-1)}(p)-f^{n(s-1)}(x)\right|>\delta-1 /(s-1)
$$

and (1.3) follows.

4.4. Proof of Theorem 2.4. Assume the trajectory of $x$ is not asymptotically periodic (otherwise (2.1) is clearly true). Then $L_{f}(x)=\omega$ is infinite. Since every two points $u, v \in \omega$ are $f$-separable, there is an $f$-periodic interval $J$ with $\omega \subset \operatorname{Orb}_{f}(J)$ and such that for every $i, \operatorname{diam}\left(f^{i}(J)\right)<\varepsilon$. Since $J$ is periodic, $J$ contains a periodic point $q$ of $f$. Choose $j$ such that $f^{j}(x) \in J$ and let $p \in \operatorname{Orb}_{f}(q)$ be such that $f^{j}(p) \in J$. Then clearly $\left|f^{n}(p)-f^{n}(x)\right|<\varepsilon$ for every $n>j$ and (2.1) is true.

4.5. Proof of Theorem 2.2. (i) $\Rightarrow$ (ii) follows from Lemma 4.3 and by Theorem 2.4 we have non-(i) $\Rightarrow$ non-(ii), since in this case, (1.3) cannot be satisfied.

5. Proof of Theorem 2.7. Examples of functions of type $2^{\infty}$ possessing infinite attractors have been indicated by Šarkovskii [14] and Kenžegulov and Šarkovskii [8]. Denote these functions by $\tilde{g}$ and $\tilde{f}$, respectively. It turns out that $\tilde{f}$ and $\tilde{g}$ actually satisfy our Theorem 2.7 , but it is rather difficult to show it. Therefore in this section 
we give another construction. Both maps $f$ and $g$ are given as the uniform limits of sequences of maps with cycles of order not greater than $2^{n+1}, n=1,2, \ldots$ The key to the construction is a general system $\left\{f_{n}\right\}$ of maps depending on some parameters. Its properties are given in Lemma 5.2. By specifying the parameters we then obtain sequences converging to $f$ or $g$, respectively.

Note that our function $g$ has similar properties as the map $\tilde{g}$ from [14], while $f$ is of quite different type than $\tilde{f}$ from [8] (or than a similar map indicated later in [25] which has zero topological entropy and seems to be chaotic, too). More precisely, both $\tilde{f}$ and the map from [25] should have infinite attractors with isolated points but $f$ has a unique infinite attractor, which is a perfect set.

We begin with the following auxiliary functions. Let $I=[a, b], K=[a, a+p]$, and $L=[b-q, b]$ be nondegenerate intervals with $K \cap L=\varnothing$. Define maps $\psi(I), \varphi(K, L) \in C^{0}(I, I)$ as follows:

$$
\psi(I)(x)=a+b-x \quad \text { for } x \in I
$$

and

$$
\varphi(K, L)(x)= \begin{cases}a & \text { for } x=a \\ a+q & \text { for } x=a+p, \\ b & \text { for } x=b-q, \\ b-p & \text { for } x=b .\end{cases}
$$

Moreover, let $\varphi(K, L)$ be linear on each of the intervals $K, L$, and $I \backslash(K \cup L)$. Denote $\tau(K, L)=\psi(I) \circ \varphi(K, L)$ (first apply $\varphi$, then $\psi$ ).

5.1. Lemma. For any disjoint, nondegenerate intervals $K, L$ with $\operatorname{conv}(K \cup L)=I$,

(i) $\tau(K, L)(K)=L$ and $\tau(K, L)(L)=K$;

(ii) $\tau^{2}(K, L)(x)=\psi(L)(x)$ for $x \in L$;

(iii) $\tau^{4}(K, L)(x)$ is the identity map for $x \in K \cup L$;

(iv) for every $y \in I \backslash(K \cup L)$, either $y$ is a (repulsive) fixed point of $\tau(K, L)$, or the trajectory of $y$ with respect to $\tau(K, L)$ is eventually in $K \cup L$.

Proof. Straightforward verification.

Now assume there is a system

$$
J_{0}^{0}=[0,1], J_{1}^{1}, J_{1}^{0}, J_{2}^{1}, J_{2}^{0}, \ldots, J_{i}^{1}, J_{i}^{0}, \ldots
$$

of closed intervals with the properties

$$
J_{i}^{1}<J_{i}^{0} \quad \text { and } \operatorname{conv}\left(J_{i}^{1} \cup J_{i}^{0}\right)=J_{i-1}^{0} \quad \text { for } i \geqslant 1
$$

$(A<B$ means $x<y$ for any $x \in A$ and $y \in B$ ). Define recursively a sequence $\left\{f_{i}\right\}_{i=0}^{\infty}$ of maps from $C^{0}([0,1],[0,1])$ :

$$
\begin{array}{ll}
f_{0}(x)=1-x & \text { for } x \in[0,1], \\
f_{k+1}=f_{k} \circ \varphi_{k} & \text { for any } k \geqslant 0,
\end{array}
$$

where

$$
\varphi_{k}(x)= \begin{cases}\varphi\left(J_{k+1}^{1}, J_{k+1}^{0}\right)(x) & \text { for } x \in J_{k}^{0} \\ x & \text { otherwise }\end{cases}
$$


5.2. LemMA. For every $k \geqslant 1$ and $i=0,1$,

(i) $f_{k}(x)=f_{j}(x)$ for $j \leqslant k$ and $x \notin J_{j}^{0}$;

(ii) $J_{k}^{i}$ is an $f_{k}$-periodic interval of order $2^{k}$;

(iii) $f_{k}^{m}(x)=\psi\left(J_{k}^{0}\right)(x)$ for $x \in J_{k}^{0}$ and $m=2^{k}$;

(iv) $f_{k}^{r}\left(J_{j}^{i}\right)=f_{j}^{r}\left(J_{j}^{i}\right)$ for $j<k$ and $r \geqslant 0$;

(v) $f_{k}^{j}$ is linear on every interval $J \subset \operatorname{Orb}_{f_{k}}\left(J_{k}^{0}\right)$ for every $j$;

(vi) $\operatorname{Orb}_{f_{k}}\left(J_{k}^{0}\right)=\operatorname{Orb}_{f_{k}}\left(J_{k}^{1}\right)$;

(vii) the $f_{k}$-trajectory of every $x \in[0,1]$ either is eventually periodic of period $\leqslant 2^{k+1}$ or is eventually in $\mathrm{Orb}_{f_{k}}\left(J_{k}^{0}\right)$.

Proof. Property (i) follows from (5.4), (5.5), and (5.2).

To show the next two properties assume by induction that (ii) and (iii) are true for $k=s$ (they are clearly true for $k=0$ ). Since $\varphi_{s}\left(J_{s}^{0}\right)=J_{s}^{0}$ and $\varphi_{s}(x)=x$ for $x \notin J_{s}^{0}$, (ii) for $k=s$ implies

$$
f_{s+1}^{j}(x)=\left(f_{s}^{j} \circ \varphi_{s}\right)(x) \text { for } x \in J_{s}^{0} \text { and } 1 \leqslant j \leqslant 2^{s} .
$$

Using (iii) for $k=s$ and (5.6) we have, for $n=2^{s}$,

$$
f_{s+1}^{n}(x)=\left(f_{s}^{n} \circ \varphi_{n}\right)(x)=\tau\left(J_{s+1}^{1}, J_{s+1}^{0}\right)(x) \text { for } x \in J_{s}^{0}
$$

and thus, by Lemma 5.1(i),

$$
f_{s+1}^{n}\left(J_{s+1}^{0}\right)=J_{s+1}^{1} \text { and } f_{s+1}^{n}\left(J_{s+1}^{1}\right)=J_{s+1}^{0} .
$$

Consequently, since $J_{s+1}^{0}$ and $J_{s+1}^{1}$ are disjoint, both these intervals are $f_{s+1}$-periodic of order $2^{s+1}$, i.e. (ii) is true for $k=s+1$.

Moreover, by (5.7), $f_{s+1}^{2 n}(x)=\tau^{2}\left(J_{s+1}^{1}, J_{s+1}^{0}\right)(x)$ for $x \in J_{s}^{0}$ and Lemma 5.1(ii) implies (iii) for $k=s+1$.

To prove (iv) it clearly suffices to show that

$$
f_{s+1}^{r}\left(J_{j}^{i}\right)=f_{s}^{r}\left(J_{j}^{i}\right) \text { for } j \leqslant s
$$

under the hypothesis that (iv) is true for $k=s$. We use induction on $r$ :

$$
f_{s+1}^{r+1}\left(J_{j}^{i}\right)=f_{s+1}\left(f_{s+1}^{r}\left(J_{j}^{i}\right)\right)=f_{s+1}\left(f_{s}^{r}\left(J_{j}^{i}\right)\right) .
$$

Since by (iv) for $k=s, f_{s}^{r}\left(J_{j}^{i}\right)=f_{j}^{r}\left(J_{j}^{i}\right)=J$ is a component interval of $\operatorname{Orb}_{f_{j}}\left(J_{j}^{i}\right)$, we have either $J_{s}^{0} \subset J$ or $J_{s}^{0} \cap J=\varnothing$ and by (5.5),

$$
f_{s+1}\left(f_{s}^{r}\left(J_{j}^{i}\right)\right)=f_{s}\left(\varphi_{s}(J)\right)=f_{s}(J)=f_{s}^{r+1}\left(J_{j}^{i}\right) .
$$

Hence (5.9) is true for $r:=r+1$.

(v) follows from (ii) and from the fact that $f_{k}$ is linear on every component interval of $\operatorname{Orb}_{f_{k}}\left(J_{k}^{0}\right)$, and (vi) follows from (ii) and (5.8).

Finally, to prove (vii) assume by induction that the condition is satisfied for $k=s$. Let $x \in[0,1]$. If $f_{s}^{j}(x) \notin \operatorname{Orb}_{f_{s}}\left(J_{s}^{0}\right)$ for every $j$, then by the hypothesis, $x$ is eventually $f_{s}$-periodic and since by (i), $f_{s+1}^{i}(x)=f_{s}^{i}(x), x$ is eventually $f_{s+1}$-periodic of period $\leqslant 2^{s+1}<2^{s+2}$.

Otherwise there is the smallest $j>0$ with $y=f_{s}^{j}(x) \in J_{s}^{0}$. Let $n=2^{s}$. Then by (5.7) and Lemma 5.1(iv), either $y$ is a fixed point or $y$ is eventually in $J_{s+1}^{1} \cup J_{s+1}^{0}$ under $f_{s+1}^{4 n}$, i.e., either $y$ is eventually in $\operatorname{Orb}_{f_{s+1}}\left(J_{s+1}^{0}\right)$ or $y$ is $f_{s+1}$-periodic of period $\leqslant 2^{s+2}$. Since by (5.4) and (5.5), $y=f_{s+1}^{j}(x)=f_{s}^{j}(x)$, (vii) is proved. 
Now we are ready to prove the easier part of Theorem 2.7.

5.3. Proof of Theorem 2.7(ii). Choose intervals (5.1) such that $\mu\left(J_{k+1}^{1}\right)=$ $\mu\left(J_{k+1}^{0}\right)=3^{-1} \mu\left(J_{k}^{0}\right)$. Then using Lemma 5.2(ii), (v), and (vi) we can easily show by induction that for every component interval $J$ of $\operatorname{Orb}_{f_{k}}\left(J_{k}^{0}\right), \mu(J)=3^{-k}$. Next using Lemma 5.2(i) and (iv), we obtain $\left\|f_{k+1}-f_{k}\right\| \leqslant 3^{-k}$ for every $k$.

Let $g=\lim _{k \rightarrow \infty} f_{k}$. Then $g$ has only cycles of order $2^{n}, n=0,1,2, \ldots$ Otherwise any $h$ from a certain neighborhood of $g$, and thus also every $f_{k}$ for sufficiently large $k$, should have a cycle of order $\neq 2^{n}$ (Block [2]) contrary to Lemma 5.2(vii).

By Lemma 5.2(iv)

$$
\operatorname{Orb}_{f_{k}}\left(J_{k}^{0}\right)=\operatorname{Orb}_{g}\left(J_{k}^{0}\right)=F_{k} .
$$

Put $F=\bigcap_{k=1}^{\infty} F_{k}$. Then $F$ is the Cantor middle-third set. Using (5.10) we see that for $x \in F$ and any $k$, any component interval of $F_{k}$ contains some $g^{j}(x)$, hence $L_{g}(x)=F$.

Moreover, $F$ is the unique infinite attractor of $g$ : If $x$ is not eventually periodic, then, for any $k, x$ is eventually in $F_{k}$ under $g$ (Lemma 5.2(vii) and (i)) hence $L_{g}(x) \subset F$. On the other hand, if $G$ is a neighborhood of a point of $F$, then $g^{i}\left(J_{j}^{0}\right) \subset G$ for some $i, j$, and hence, for a suitable $s, g^{s}(x) \in G$. Thus $L_{g}(x)=F$.

Finally, let $x, y \in F, x \neq y$. Then $x, y$ belong to different component intervals of $F_{k}$ for any $k$ with $3^{-k}<|x-y|$. By Lemma 5.2(ii), $x$ and $y$ are $g$-separable and Theorem 2.2 now implies that $g$ is not chaotic in the sense of $\mathrm{Li}$ and Yorke.

5.4. Proof of Theorem 2.7(i). First we show that the intervals (5.1) can be chosen such that for every $k$,

$$
F_{k} \text { contains a component interval } I_{k} \text { with } \mu\left(I_{k}\right)>1 / 2 \text {, }
$$

where $F_{k}=\operatorname{Orb}_{f_{k}}\left(J_{k}^{0}\right)$. Clearly (5.11) is true for $k=0$. Assume by induction (5.11) is true for a certain $k$. Take $n$ such that $f_{k}^{n}\left(I_{k}\right)=J_{k}^{0}$ (Lemma 5.2(ii)) and let $K_{k}$, $L_{k} \subset I_{k}$ be disjoint closed intervals with $\operatorname{conv}\left(K_{k} \cup L_{k}\right)=I_{k}$. Denote $f_{k}^{n}\left(K_{k}\right)=$ $J_{k+1}^{1}$ and $f_{k}^{n}\left(L_{k}\right)=J_{k+1}^{0}$. By Lemma 5.2(v) a suitable choice of $K_{k}, L_{k}$ (i.e. $K_{k}<L_{k}$ or $L_{k}<K_{k}$ ) implies (5.2) for $i=k+1$.

If we take $K_{k}, L_{k}$ such that one of the conditions

$$
\mu\left(K_{k}\right)>1 / 2 \text { or } \mu\left(L_{k}\right)>1 / 2
$$

is satisfied, then clearly (5.11) is true for $k:=k+1$.

Thus we have defined $J_{k+1}^{1}, J_{k+1}^{0}$ recursively from $f_{k}$ and $J_{k}^{0}$. Since by (5.3)-(5.5), $f_{k+1}$ is defined recursively from $f_{k}, J_{k+1}^{1}$, and $J_{k+1}^{0}$, this modification leaves Lemma 5.2 true for arbitrary choice of parameters $\mu\left(K_{k}\right)$ and $\mu\left(L_{k}\right)$ satisfying (5.12). Next we shall specify how to choose the parameters.

Denote $\rho_{k}=\mu\left(f_{k}\left(J_{k}^{0}\right)\right) / \mu\left(J_{k}^{0}\right)$. Since $\mu\left(\varphi_{k}\left(J_{k}^{0}\right)\right)=\mu\left(J_{k}^{1}\right)$ (see (5.5)), Lemma 5.2(v) gives

$$
\mu\left(f_{k+1}\left(J_{k+1}^{0}\right)\right)=\mu\left(f_{k}\left(\varphi_{k}\left(J_{k+1}^{0}\right)\right)\right)=\mu\left(f_{k}\left(J_{k+1}^{1}\right)\right)=\rho_{k} \mu\left(J_{k+1}^{1}\right) .
$$

Moreover, there is an $\varepsilon_{k}>0$ such that

$$
\mu\left(K_{k}\right)<\varepsilon_{k} \Rightarrow \rho_{k} \mu\left(J_{k+1}^{1}\right)=\rho_{k} \mu\left(f_{k}^{n}\left(K_{k}\right)\right)<\frac{1}{2} \mu\left(f_{k}\left(J_{k}^{0}\right)\right) .
$$


Now if we choose $K_{k}, L_{k}$ such that

$$
\mu\left(L_{k}\right)>\frac{1}{2} \text { and } \mu\left(K_{k}\right)<\varepsilon_{k} \text { for any } k \text { odd, }
$$

then (since $\left.f_{k+1}\left(J_{k+1}^{0}\right) \subset f_{k}\left(J_{k}^{0}\right)\right)(5.13)$ and (5.14) imply

$$
\sum_{k=1}^{\infty} \mu\left(f_{k}\left(J_{k}^{0}\right)<\infty\right. \text {. }
$$

Since by Lemma 5.2(i),

$$
\begin{aligned}
\left\|f_{k+1}-f_{k}\right\| & =\max \left\{\left|f_{k+1}-(x)-f_{k}(x)\right| ; x \in J_{k}^{0}\right\} \\
& =\max \left\{\left|f_{k}\left(\varphi_{k}(x)\right)-f_{k}(x)\right| ; x \in J_{k}^{0}\right\} \\
& \leqslant \mu\left(f_{k}\left(J_{k}^{0}\right)\right),
\end{aligned}
$$

(5.16) implies that $\lim _{k \rightarrow \infty} f_{k}=f$ uniformly.

Denote $F=\bigcap_{k=1}^{\infty} F_{k}$, and let $J=[u, v]$ be the connected component of $F$ with $\mu(J) \geqslant 1 / 2$ (see (5.11)). In the sequel we will need the following condition to be satisfied:

Any neighborhood of either of the points $u, v$ contains a component interval from a certain $F_{i}$.

This condition is clearly satisfied, when we choose the parameters $\mu\left(K_{k}\right), \mu\left(L_{k}\right)$ for the even $k$ 's (see (5.12) and (5.15)) as follows:

$$
\mu\left(L_{k}\right)>1 / 2 \text { if } \begin{cases}K_{k}<L_{k} & \text { and } k \equiv 0(\bmod 4) \text { or } \\ L_{k}<K_{k} & \text { and } k \equiv 2(\bmod 4)\end{cases}
$$

and $\mu\left(K_{k}\right)>1 / 2$ (and so $\mu\left(L_{k}\right)<1 / 2$ ) otherwise.

Now let $U$ be a connected component of $F$. For any $k$, take $U_{k}$ to be the component interval of $F_{k}$ with $U \subset U_{k}$. Then by Lemma 5.2(iv) and (ii), $f^{i}(U) \subset$ $f^{i}\left(U_{k}\right)=f_{k}^{i}\left(U_{k}\right)$ and $f_{k}^{i}\left(U_{k}\right) \cap U_{k}=\varnothing$ for $i<2^{k}$. Consequently,

$$
f^{i}(U) \cap U=\varnothing \quad \text { for any } i>0 \text {. }
$$

Let $y \in J=[u, v]$. Then for any $k$, every component interval of $F_{k}$ contains infinitely many members of the sequence $\left\{f^{i}(y)\right\}_{i=1}^{\infty}$ and hence $\omega=L_{f}(y)$ is infinite. Moreover, by (5.17) any neighborhood of $u$ contains some $f^{i}(y)$ and hence $u \in \omega$. Similarly, $v \in \omega$.

Clearly, $f$ is of type $2^{\infty}$ (the argument is similar to the one in the proof of Theorem 2.7(ii)). Thus by Theorem 2.2 to finish the proof it suffices to show that $u, v \in \omega$ are $f$-nonseparable.

Assume the contrary. Let $J_{u}, J_{v}$ be disjoint $f$-periodic intervals of order $\leqslant 2^{k}=m$ with $u \in J_{u}, v \in J_{v}$. Then by (5.18) either $f^{m}(J)<J$ or $J<f^{m}(J)$. Assume, e.g., $f^{m}(J)<J$. Then $J_{v}=f^{m}\left(J_{v}\right) \supset \operatorname{conv}\left\{v, f^{m}(v)\right\} \supset[u, v]$, i.e., $u \in J_{v}$-a contradiction.

5.5. REMARK. It turns out that the map $f$ from the above proof has homtervals (in the sense of Misiurewicz), i.e. intervals, all of whose images are pairwise disjoint and mapped monotonically by any iterate of $f$. Keeping the notation from the proof, one can easily see that, e.g., $J=[u, v]$ is such an interval. 
On the other hand, clearly the map $g$ from the proof of Theorem 2.7(ii) has no homtervals. This would suggest that the existence of homtervals is connected with the chaoticity of maps of type $2^{\infty}$. But this is not the case; the existence of homtervals is irrelevant for the chaoticity. (One can only prove that any chaotic map of type $2^{\infty}$ must have a wandering interval attracted by an infinite set. For nonchaotic mappings this statement is not true.)

Actually there are mappings $f^{*}, g^{*} \in C^{0}([0,1],[0,1])$ both with zero topological entropy and infinite attractors such that $f^{*}$ is chaotic and has no homtervals and $g^{*}$ is not chaotic but has homtervals. The mapping $f^{*}$ can be obtained by a slight modification of our map $f$ from the proof of Theorem 2.7(i):

Let $H=\left\{U_{n}\right\}_{n=1}^{\infty}$ be the system of maximal homtervals of $f$. Put $f^{*}(x)=f(x)$ for $x \in[0,1] \backslash \bigcup_{n=1}^{\infty} U_{n}$, and let $f^{*}\left(U_{n}\right)=f\left(U_{n}\right)$ for any $n$, and $f^{*}$ be monotonic on no subinterval of $U_{n}$. It is easy to verify that $f^{*}$ has the desired properties.

As an example of $g^{*}$ one can probably take Harrison's mapping (cf. [6]), but the proof seems to be difficult. So we provide an outline of another construction, modifying simply our map $g$ from the proof of Theorem 2.7(ii).

5.6. EXAMPLE. Keep the notation from the proof of Theorem 2.7(ii). Denote by $I_{n}$ the (closed) interval such that each of the sets $I_{n} \cap J_{n}^{0}$ and $I_{n} \cap J_{n}^{1}$ contains just one point, and let $K_{n}$ be a (nontrivial) closed interval lying in the interior of $I_{n}$, $n=1,2, \ldots$.

Clearly, $g$ maps $I_{1}$ monotonically onto $I_{1} \cup J_{1}^{0}$. Let $g_{1} \in C^{0}([0,1],[0,1])$ be such that $g_{1}(x)=g(x)$ for $x \notin I_{1}$, and let $g_{1}$ be strictly monotonic on $I_{1}$ such that $g_{1}\left(I_{1}\right)=I_{1} \cup J_{1}^{0}$ and $g_{1}\left(K_{1}\right)=K_{2}$. We can easily see that $g_{1}$ has properties similar to $g$ (i.e. $g_{1}$ is not chaotic, has a unique infinite attractor - the Cantor set, and any point from $K_{1}$ is attracted to it).

Similarly, we put $g_{2}(x)=g_{1}(x)$ for $x \notin I_{2}$, and set $g_{2}$ monotonic on $I_{2}$ with $g_{2}^{2}\left(I_{2}\right)=I_{2} \cup J_{2}^{0}\left(=g^{2}\left(I_{2}\right)\right)$ and $g_{2}^{2}\left(K_{2}\right)=K_{3}$. Next, changing $g_{2}$ appropriately on the interval $I_{3}$, we obtain $g_{3}$, etc. Clearly, $\lim _{n \rightarrow \infty} g_{n}=g^{*}$ uniformly and $g^{*}$ is the desired mapping: It is nonchaotic and $K_{1}$ is its homterval attracted by the Cantor set (we have $g^{* 2^{n}}\left(K_{1}\right)=K_{n+1}$ for any $n$ ).

ACKnowledgment. I would like to thank to Professor A. N. Šarkovskii for stimulating discussions during the 1st Czechoslovak Summer School on Dynamical Systems in June 1984.

\section{REFERENCES}

1. L. Block, Homoclinic points of mappings of the interval, Proc. Amer. Math. Soc. 72 (1978), 576-580.

2. Stability of periodic orbits in the theorem of Šarkuvskii, Proc. Amer. Math. Soc. 82 (1981), $333-336$.

3. G. J. Butler and G. Pianigiani, Periodic points and chaotic functions in the unit interval, Bull. Austral. Math. Soc. 18 (1978), 255-265.

4. W. A. Coppel, Maps of an interval, Preprint, Univ. of Minnesota, 1983.

5. R. Graw, On the connection between periodicity and chaos of continuous functions and their iterates, Aequationes Math. 19 (1979), 277-278.

6. J. Harrison, Wandering intervals, Dynamical Systems and Turbulence (Warwick, 1980), Lecture Notes in Math., vol. 898, Springer, Berlin, Heidelberg and New York, 1981, pp. 154-163.

7. I. Kan, A chaotic function possessing a scrambled set with positive Lebesgue measure, Proc. Amer. Math. Soc. 92 (1984), 45-49. 
8. Ch. K. Kenžegulov and A. N. Šarkovskii, On properties of the set of limit points of an iterated sequence of a continuous function, Volž. Mat. Sb. 3 (1965), 343-348. (Russian)

9. L. Kuipers and L. Niederreiter, Uniform distribution of sequences, Wiley, New York, 1974, p. 8.

10. C. Kuratowski, Topologie. I, PWN, Warsaw, 1958.

11. T. Y. Li and J. A. Yorke, Period three implies chaos, Amer. Math. Monthly 82 (1975), 985-992.

12. M. Misiurewicz, Horseshoes for mappings of the interval, Bull. Acad. Polon. Sci. Sér. Math. 27 (1979), 167-169.

13. Chaos almost everywhere, Preprint, 1983.

14. A. N. Šarkovskii, Co-existence of cycles of continuous maps of the line into itself, Ukrain. Mat. Ž. 16 (1964), 61-71. (Russian)

15. On cycles and the structure of continuous mappings, Ukrain. Mat. Ž. 17 (1965), 104-111. (Russian)

16. The behavior of a map in a neighborhood of an attracting set, Ukrain. Mat. Ž. 18 (1966), 60-83. (Russian)

17. _ The partially ordered system of attracting sets, Soviet Math. Dokl. 7 (1966), 1384-1386.

18. __ Attracting sets containing no cycles, Ukrain. Mat. Ž. 20 (1968), 136-142. (Russian)

19. On some properties of discrete dynamical systems, Proc. Internat. Colloq. on Iteration Theory and its Applications, Toulouse, 1982.

20. J. Smital and K. Smitalová, Structural stability of nonchaotic difference equations, J. Math. Anal. Appl. 90 (1982), 1-11; Errata 101 (1984), p. 324.

21. J. Smital, A chaotic function with some extremal properties, Proc. Amer. Math. Soc. 87 (1983), 54-56. 22. , A chaotic function with a scrambled set of positive Lebesgue measure, Proc. Amer. Math.

Soc. 92 (1984), 50-54.

23. J. Smital and K. Janková, Characterization of chaos, Bull. Austral. Math. Soc. (to appear).

24. P. Štefan, $A$ theorem of Šarkouskii on existence of periodic orbits of a continuous endomorphisms of the real line, Comm. Math. Phys. 54 (1977), 237-248.

25. M. B. Verejkina and A. N. Šarkovskii, Recurrence in one-dimensional dynamical systems, Approx. and Qualitative Methods of the Theory of Differential-Functional Equations, Inst. Math. Akad. Nauk USSR, Kiev, 1983, pp. 35-46. (Russian)

Department of Mathematics, Komensky University, 84215 Bratislava, Czechoslovakia 